\title{
Universiteit
}

Leiden

The Netherlands

\section{Temperature-dependent third cumulant of tunneling noise}

Beenakker, C.W.J.; Kindermann, M.; Nazarov, Yu.V.

\section{Citation}

Beenakker, C. W. J., Kindermann, M., \& Nazarov, Y. V. (2003). Temperature-dependent third cumulant of tunneling noise. Retrieved from https://hdl.handle.net/1887/1283

Version: $\quad$ Not Applicable (or Unknown)

License: $\quad$ Leiden University Non-exclusive license

Downloaded from: https://hdl.handle.net/1887/1283

Note: To cite this publication please use the final published version (if applicable). 


\title{
Temperature-Dependent Third Cumulant of Tunneling Noise
}

\author{
C W J Beenakkeı, ${ }^{1} \mathrm{M}$ Kındeımann, ${ }^{1}$ and Yu V Nazaı ${ }^{2}$ \\ 'Instutuut Lorentz Universiteit Leiden, PO Box 9506, 2300 RA Leiden The Netherlands \\ ${ }^{2}$ Department of Nanoscience, Delft University of Technology Lorentzweg 1, 2628 CJ Delft The Netherlands
}

(Received 24 Januaty 2003, published 28 Apil 2003)

\begin{abstract}
Poisson statistics predicts that the shot noise in a tunnel junction has a temperature independent third cumulant $e^{2} \bar{I}$, deter mined solely by the mean cur ent $\bar{I}$ Experimental data, howeve1, show a puzzling temperature dependence We demonstrate theoretically that the thud cumulant becomes stiongly temperature dependent and may even change sign as a result of feedback from the electromagnetic environment In the limit of a noninvasive (zeio impedance) measurement circuit in thei mal equilib 11 m with the junction, we find that the thisd cumulant ciosses over fiom $e^{2} \bar{I}$ at low temperatures to $-e^{2} \bar{I}$ at high temperatures
\end{abstract}

\section{DOI 10 1103/PhysRevLett 90176802}

Shot noise of the electical cur rent was studied a century ago as a way to measuie the fundamental unit of charge [1] Today shot noise is used for this puipose in a wide iange of contexts, including superconductivity and the fractional quantum Hall effect [2] Alieady in the earliest work on vacuum tubes it was realized that thermal fluctuations of the curient can mask the fluctuations due to the discreteness of the chaige In semiconductors, in particula1, accurate measurements of shot norse are notollously difficult because of the 1equirement to maintain a low temperature at a high applied voltage

Until very recently, only the second cumulant of the fluctuatıng cuirent was ever measured The distribution of transferied chaige is nearly Gaussian, because of the law of laige numbers, so it is quite nontrivial to extract cumulants highet than the second Much of the exper1 mental effort was motivated by the piediction of Levitov and Reznikov [3] that odd cumulants of the cuitent thiough a tunnel junction should not be affected by the theimal norse that contaminates the even cumulants This is a direct consequence of the Poisson statistics of tunnelıng events The thind cumulant should thus have the linear dependence on the applied voltage char acter 1stic of shot norse, 1egardless of the ratio of voltage and temperature In contrast, the second cumulant levels off at the ther mal norse for low voltages

The first expe1iments on the voltage dependence of the thi1d cumulant of tunnel noise have now been reported [4] The pictures are stiıkingly different fiom what was expected theoretically The slope varies by an order of magnitude between low and high voltages, and for ceitain samples even changes sign Such a behaviol is expected for a diffusive conductor [5], but not for a tunnel junction Although the data are st11l preliminar $y$, it seems clear that an input of new physics is 1equired for an undeistanding It ts the puipose of this paper to provide such input

We will show that the thin cumulant of the measured noise (unlike the second cumulant [6]) is affected by the measurement cricurt in a nonlinear way The effect can be seen as a backaction of the electiomagnetic envisonment
PACS numbers $7350 \mathrm{Td}, 0540 \mathrm{Ca} 7270+\mathrm{m} 7440+\mathrm{k}$

[7] We have found that the backaction peisists even in the limit of zeio impedance, when the measurement is supposed to be noninvasive The temperature independent result for the thind cumulant of tunneling norse is recovered only if the measurement cucult has both negligible impedance and negligible temperature

The circult is shown schematically in Fig 1 Two 1esistors (1mpedances $Z_{1}, Z_{2}$ and temperatures $T_{1}, T_{2}$ ) are connected in series to a voltage source (voltage $V_{0}$ ) We will specialize later to the case that iesistor 1 is a tunnel junction and that resistor 2 represents the macioscopic measurement circuit, but our main results hold for any two resistors We disiegard possible Coulomb blockade effects on fluctuations [8-10], which is justıfied if the impedances at frequencies of order $\mathrm{eV} / \hbar$ are small compared to $\hbar / e^{2}[11]$

We have calculated the temperature dependence of the third cumulant by two altogether different methods, the Keldysh formalısm [12] and the Langevin approach [13] The equivalence of the two methods has alieady been demonstrated for a single resistor in the absence of any

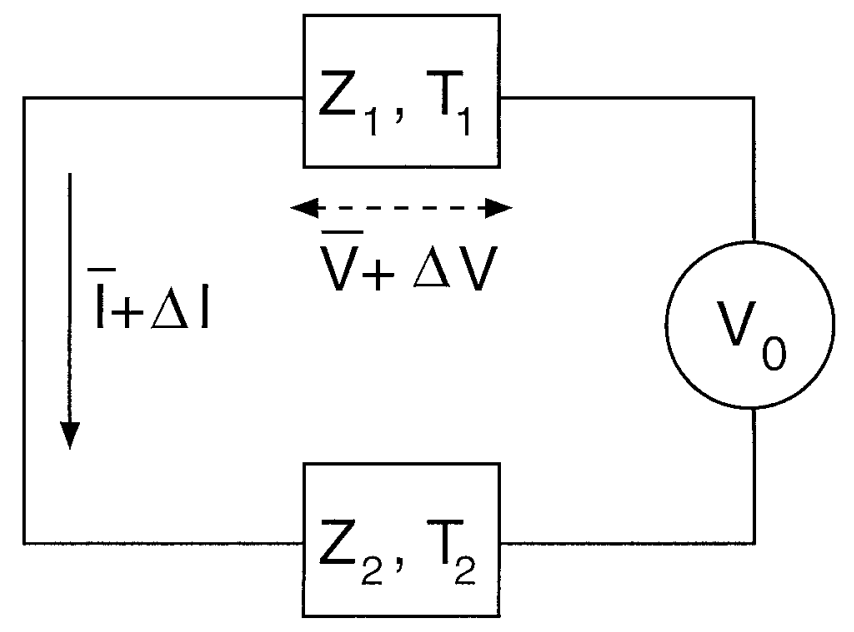

FIG 1 Two resistors in sciles wilh a voltage source The fluctuating curient and voltage are indicated 
measurement circuit [14]. Likewise, we have obtained the same results in both calculations of the backaction from the measurement. We choose to present the Langevin approach in this Letter, because it can be explained in elementary terms and provides an intuitive physical insight.

The starting point of the Langevin approach is the separation of the fluctuation $\Delta I_{l}$ of the current through resistor $i=1,2$ into an intrinsic fluctuation $\delta I_{t}$ plus a term induced by a fluctuation $\Delta V_{l}$ of the voltage over the resistor: $\Delta I_{l}=\delta I_{l}+\Delta V_{l} / Z_{l}$. At low frequencies $\Delta I_{1}=$ $\Delta I_{2} \equiv \Delta I$ and $\Delta V_{1}=-\Delta V_{2} \equiv \Delta V$. Upon substitution we arrive at the two equations

$$
Z \Delta I=Z_{1} \delta I_{1}+Z_{2} \delta I_{2}, \quad Z \Delta V=Z_{1} Z_{2}\left(\delta I_{2}-\delta I_{1}\right)
$$

where $Z=Z_{1}+Z_{2}$ is the total impedance of the circuit.

For simplicity we assume that $Z_{l}$ is real and frequency independent in the frequency range of the measurement. All formulas have a straightforward generalization to complex $Z_{t}(\omega)$. We do not need to assume at this stage that the current-voltage characteristic of the resistors is linear. If it is not, then one should simply replace $1 / Z_{l}$ by the differential conductance evaluated at the mean voltage $\bar{V}_{l}$ over the resistor.

The mean voltages are given by $\bar{V}_{1}=\left(Z_{1} / Z\right) V_{0} \equiv \bar{V}$ and $\bar{V}_{2}=V_{0}-\bar{V}$. The intrinsic current fluctuations $\delta I_{t}$ are driven by the fluctuating voltage $V_{1}=\bar{V}_{l}+\Delta V_{1}$, and therefore depend in a nonlinear way on $\Delta V$. The nonlinearity has the effect of mixing in lower order cumulants of $\delta I_{t}$ in the calculation of the $p$ th cumulant of $\Delta I$, starting from $p=3$.

Before addressing the case $p=3$ we first consider $p=2$, when all averages $\langle\cdots\rangle_{\bar{V}}$ can be performed at the mean voltage. At low frequencies one has

$$
\left\langle\delta I_{\imath}(\omega) \delta I_{l}\left(\omega^{\prime}\right)\right\rangle_{\bar{V}}=2 \pi \delta\left(\omega+\omega^{\prime}\right) C_{t}^{(2)}\left(\bar{V}_{\imath}\right)
$$

The noise power $C_{l}^{(2)}$ depends on the model for the resistor. We give two examples. In a macroscopic resistor the shot noise is suppressed by electron-phonon scattering and only thermal noise remains:

$$
C_{l}^{(2)}=2 k T_{l} / Z_{l}
$$

at temperature $T_{1}$, independent of the voltage. (The noise power is a factor of 2 larger if positive and negative frequencies are identified.) In a tunnel junction both thermal noise and shot noise coexist, according to [2]

$$
C_{l}^{(2)}\left(\bar{V}_{l}\right)=\left(e \bar{V}_{l} / Z_{l}\right) \operatorname{coth}\left(e \bar{V}_{l} / 2 k T_{l}\right)
$$

From Eq. (1) we compute the correlator

$$
\left\langle\Delta X(\omega) \Delta Y\left(\omega^{\prime}\right)\right\rangle_{\bar{V}}=2 \pi \delta\left(\omega+\omega^{\prime}\right) S_{X Y}(\bar{V}),
$$

where $X$ and $Y$ can represent $I$ or $V$. The result is

$$
\begin{aligned}
S_{I I} & =Z^{-2}\left[Z_{1}^{2} C_{1}^{(2)}(\bar{V})+Z_{2}^{2} C_{2}^{(2)}\left(V_{0}-\bar{V}\right)\right], \\
S_{V V} & =Z^{-2}\left(Z_{1} Z_{2}\right)^{2}\left[C_{1}^{(2)}(\bar{V})+C_{2}^{(2)}\left(V_{0}-\bar{V}\right)\right], \\
S_{I V} & =Z^{-2} Z_{1} Z_{2}\left[Z_{2} C_{2}^{(2)}\left(V_{0}-\bar{V}\right)-Z_{1} C_{1}^{(2)}(\bar{V})\right] .
\end{aligned}
$$

Equation (5) applies to a time independent mean voltage $\bar{V}$. For a time dependent perturbation $v(t)$ one has, to linear order,

$$
\begin{aligned}
\left\langle\Delta X(\omega) \Delta Y\left(\omega^{\prime}\right)\right\rangle_{\bar{V}+v}= & \left\langle\Delta X(\omega) \Delta Y\left(\omega^{\prime}\right)\right\rangle_{\bar{V}} \\
& +v\left(\omega+\omega^{\prime}\right) \frac{d}{d \bar{V}} S_{X Y}(\bar{V}) .
\end{aligned}
$$

We will use this equation, with $v=\Delta V$, to describe the effect of a fluctuating voltage over the resistors. This assumes a separation of time scales between $\Delta V$ and the intrinsic current fluctuations $\delta I_{l}$, so that we can first average over $\delta I_{l}$ for given $\Delta V$ and then average over $\Delta V$.

Turning now to the third cumulant, we first note that at fixed voltage the intrinsic current fluctuations $\delta I_{1}$ and $\delta I_{2}$ are uncorrelated, with third moment

$$
\begin{aligned}
\left\langle\delta I_{l}\left(\omega_{1}\right) \delta I_{l}\left(\omega_{2}\right) \delta I_{t}\left(\omega_{3}\right)\right\rangle_{\bar{V}}= & 2 \pi \delta\left(\omega_{1}+\omega_{2}+\omega_{3}\right) \\
& \times C_{i}^{(3)}\left(\bar{V}_{t}\right) .
\end{aligned}
$$

The spectral density $C_{l}^{(3)}$ vanishes for a macroscopic resistor. For a tunnel junction it has the temperature independent value [3]

$$
C_{l}^{(3)}\left(\bar{V}_{\imath}\right)=e^{2} \bar{V}_{l} / Z_{\imath}=e^{2} \bar{I}
$$

with $\bar{I}$ the mean current.

We introduce the nonlinear feedback from the voltage fluctuations through the relation

$$
\begin{aligned}
\left\langle\Delta X_{1} \Delta X_{2} \Delta X_{3}\right\rangle= & \left\langle\Delta X_{1} \Delta X_{2} \Delta X_{3}\right\rangle_{\bar{V}} \\
& +\sum_{\text {cychc }}\left\langle\Delta X_{l} \Delta V\left(\omega_{k}+\omega_{l}\right)\right\rangle_{\bar{V}} \frac{d}{d \bar{V}} \\
& \times S_{X_{k} X_{l}}(\bar{V}) .
\end{aligned}
$$

The variable $X_{l}$ stands for $I\left(\omega_{j}\right)$ or $V\left(\omega_{1}\right)$ and the sum is over the three cyclic permutations $j, k, l$ of the indices $1,2,3$. These three terms account for the fact that the same voltage fluctuation $\Delta V$ that affects $S_{X_{k} X_{Y}}$ also correlates with $X_{j}$, resulting in a cross correlation.

Equation (10) has the same form as the "cascaded average" through which Nagaev introduced a nonlinear feedback into the Langevin equation [13]. In that work the nonlinearity appears because the Langevin source depends on the electron density, which is itself a fluctuating quantity - but on a slower time scale, so the averages can be carried out separately, or "cascaded." In our case the voltage drop $\Delta V_{r}$ over the resistors is the slow variable, relative to the intrinsic current fluctuations $\delta I_{t}$.

Equation (10) determines the current and voltage correlators 


$$
\left\langle\Delta X\left(\omega_{1}\right) \Delta Y\left(\omega_{2}\right) \Delta Z\left(\omega_{3}\right)\right\rangle=2 \pi \delta\left(\omega_{1}+\omega_{2}+\omega_{3}\right) C_{X Y Z}(\bar{V})
$$

We find

$$
\begin{aligned}
C_{I I I} & =Z^{-3}\left[Z_{1}^{3} C_{1}^{(3)}(\bar{V})+Z_{2}^{3} C_{2}^{(3)}\left(V_{0}-\bar{V}\right)\right]+3 S_{I V} \frac{d}{d \bar{V}} S_{I I} \\
C_{V V V} & =Z^{-3}\left(Z_{1} Z_{2}\right)^{3}\left[C_{2}^{(3)}\left(V_{0}-\bar{V}\right)-C_{1}^{(3)}(\bar{V})\right]+3 S_{V V} \frac{d}{d \bar{V}} S_{V V}, \\
C_{V V I} & =Z^{-3}\left(Z_{1} Z_{2}\right)^{2}\left[Z_{1} C_{1}^{(3)}(\bar{V})+Z_{2} C_{2}^{(3)}\left(V_{0}-\bar{V}\right)\right]+2 S_{V V} \frac{d}{d \bar{V}} S_{I V}+S_{I V} \frac{d}{d \bar{V}} S_{V V}, \\
C_{I I V} & =Z^{-3} Z_{1} Z_{2}\left[Z_{2}^{2} C_{2}^{(3)}\left(V_{0}-\bar{V}\right)-Z_{1}^{2} C_{1}^{(3)}(\bar{V})\right]+2 S_{I V} \frac{d}{d \bar{V}} S_{I V}+S_{V V} \frac{d}{d \bar{V}} S_{I I}
\end{aligned}
$$

We apply the general result (12) to a tunnel banıer (iesistor number 1) in selies with a macroscopic resistor (number 2) The spectial densities $C_{1}^{(2)}$ and $C_{1}^{(3)}$ are given by Eqs (4) and (9), 1espectively For $C_{2}^{(2)}$ we use Eq (3), while $C_{2}^{(3)}=0$ Fiom this point on we assume lineai cur ent-voltage characteristics, so $\bar{V}$-independent $Z_{l}$ 's We compare $C_{I} \equiv C_{I I I}$ with $C_{V} \equiv-C_{V V V} / Z_{2}^{3}$ The choice of $C_{V}$ is motivated by the typical experimental situation in which one measuies the cuivent fluctuations indirectly through the voltage over a mactoscopic series resistor Fiom Eq (12) we find

$$
\begin{aligned}
C_{x}= & \frac{e^{2} \bar{I}}{\left(1+Z_{2} / Z_{1}\right)^{3}} \\
& \times\left[1+\frac{3(\sinh u \cosh u-u)}{\left(1+Z_{1} / Z_{2}\right) \sinh ^{2} u}\left(\frac{T_{2}}{T_{1}} \frac{g_{x}}{u}-\operatorname{coth} u\right)\right],
\end{aligned}
$$

with $g_{I}=1, g_{V}=-Z_{1} / Z_{2}$, and $u=e \bar{V} / 2 k T_{1}$

In the shot noise limit $\left(e \bar{V} \gg k T_{1}\right)$ we recover the thind cumulant obtained in Ref [7] by the Keldysh technique

$$
C_{I}=C_{V}=e^{2} \bar{I} \frac{1-2 Z_{2} / Z_{1}}{\left(1+Z_{2} / Z_{1}\right)^{4}}
$$

In the opposite $11 \mathrm{~m} 1 \mathrm{t}$ of small voltages $\left(e \bar{V} \ll k T_{1}\right)$ we obtain

$$
\begin{gathered}
C_{I}=e^{2} \bar{I} \frac{1+\left(Z_{2} / Z_{1}\right)\left(2 T_{2} / T_{1}-1\right)}{\left(1+Z_{2} / Z_{1}\right)^{4}}, \\
C_{V}=e^{2} \bar{I} \frac{1-Z_{2} / Z_{1}-2 T_{2} / T_{1}}{\left(1+Z_{2} / Z_{1}\right)^{4}}
\end{gathered}
$$

We conclude that there is a change in the slope $d C_{v} / d \bar{I}$ fiom low to high voltages If the ent11e system is in ther mal equilibilum $\left(T_{2}=T_{1}\right)$, then the change in slope is a factor $\pm\left(Z_{1}-2 Z_{2}\right)\left(Z_{1}+Z_{2}\right)^{-1}$, where the + sign is for $C_{I}$ and the - sign for $C_{V}$ In Fig 2 we plot the entire voltage dependence of the thind cumulants

The limit $Z_{2} / Z_{1} \rightarrow 0$ of a noninvasive measuiement is of particular interest Then $C_{I}=e^{2} \bar{l}$ has the expected result for an isolated tunnel junction [3], but $C_{V}$ remains affected by the measurement cucult

$$
\lim _{Z_{2} / Z_{1} \rightarrow 0} C_{V}=e^{2} \bar{I}\left(1-\frac{T_{2}}{T_{1}} \frac{3(\sinh u \cosh u-u)}{u \sinh ^{2} u}\right)
$$

This limit is also plotted in Fig 2, for the case $T_{2}=T_{1} \equiv$ $T$ of thermal equilibirum between the tunnel junction and the macioscopic series iesistor The slope then changes from $d C_{V} / d \bar{I}=-e^{2}$ at low voltages to $d C_{V} / d \bar{I}=e^{2}$ at high voltages The minımum $C_{V}=-17 e k T / Z_{1}=$ $-06 e^{2} \bar{I}$ is reached at $e \bar{V}=27 k T$

In conclusion, we have demonstiated that feedback from the measurement cilcult introduces a temperature dependence of the thisd cumulant of tunneling norse The

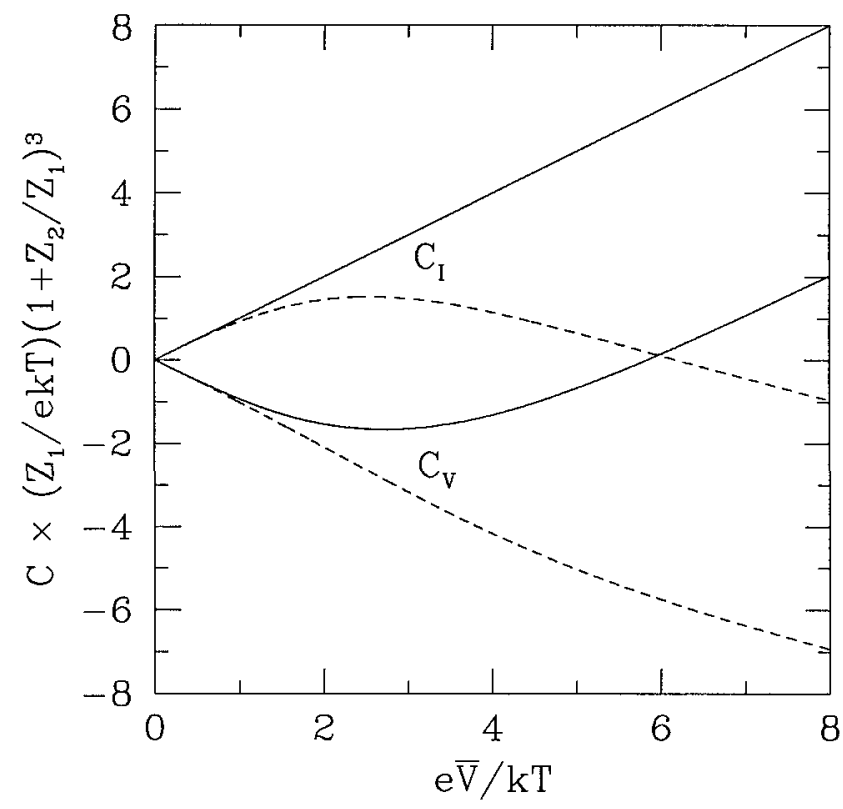

FIG 2 Voltage dependence of the thi1d cumulants $C_{l}$ and $C_{1}$ of curtent and voltage for a tunnel junction (iesistance $Z_{1}$ ) in series with a macioscopic iesistor $Z_{2}$ The two solid curves ale for $Z_{2} / Z_{1} \rightarrow 0$ and the dashed curves for $Z_{2} / Z_{1}=1$ The culves are computed from Eq (13) for $T_{1}=T_{2} \equiv T$ The high voltage slopes are the same for $C_{l}$ and $C_{1}$, while the low voltage slopes have the opposite sign 
temperature independent result $e^{2} \bar{I}$ of an isolated tunnel junction [3] acquires a striking temperature dependence in an electromagnetic environment, to the extent that the third cumulant may even change its sign. Precise predictions have been made for the dependence of the noise on the environmental impedance and temperature, which can be tested in ongoing experiments [4].

We gratefully acknowledge discussions with B. Reulet, which motivated us to write this Letter. Our research is supported by the Dutch Science Foundation NWO/FOM.

Note added-For a comparison of our theory with experimental data, see Reulet, Senzier, and Prober [15].

[1] W. Schottky, Ann. Phys. (Leipzig) 57, 541 (1918); 68, 157 (1922).

[2] Ya. M. Blanter and M. Büttiker, Phys. Rep. 336, 1 (2000).

[3] L. S. Levitov and M. Reznikov, cond-ma1/0111057.

[4] Experimental data by B. Reulet et al., contribution to the International Workshop on "Electrons in ZeroDimensional Conductors," Max Planck Institute for the Physics of Complex Systems, Dresden, 2002.
[5] D. B. Gutman and Y. Gefen, cond-mat/0201007.

[6] U. Gavish, Y. Imry, Y. Levinson, and B. Yurke, cond-mat/ 0211646.

[7] M. Kindermann, Yu. V. Nazarov, and C.W. J. Beenakker, cond-mat/0210617.

[8] E. Ben-Jacob, E. Mottola, and G. Schön, Phys. Rev. Lett. 51, 2064 (1983); G. Schön, Phys. Rev. B 32, 4469 (1985).

[9] H. Lee and L. S. Levitov, Phys. Rev. B 53, 7383 (1996).

[10] A.V. Galaktionov, D. S. Golubev, and A. D. Zaikin, condmat/0212494.

[11] G.-L. Ingold and Yu. V. Nazarov, in Single Charge Tunneling, edited by $\mathrm{H}$. Grabert and M. H. Devoret, NATO ASI, Ser. B, Vol, 294 (Plenum, New York, 1992).

[12] Yu.V. Nazarov, Ann. Phys. (Leipzig) 8, 507 (1999); Yu.V. Nazarov and M. Kindermann, cond-mat/0107133.

[13] K. E. Nagaev, Phys. Rev. B 66, 075334 (2002); K. E. Nagaev, P. Samuelsson, and S. Pilgram, Phys. Rev. B 66, 195318 (2002); S. Pilgram, A. N. Jordan, E.V. Sukhorukov, and M. Büttiker, cond-mat/0212446.

[14] D. B. Gutman, Y. Gefen, and A. D. Mirlin, cond-mat/ 0210076.

[15] B. Reulet, J. Senzier, and D. E. Prober, cond-mat/ 0302084. 